\title{
Tunneling Nanotube-Mediated Mitochondrial Transfer: A New Approach to Cell Protection
}

\author{
Aydın Bölük ${ }^{1}$ Ceren Bilgi², Eda Kutlư ${ }^{1}$, Dilek Akbaş ${ }^{2}$ and Tuba Gökdoğan Edgünlü ${ }^{3 *}$ \\ ${ }^{1}$ Faculty of Medicine, Muğla Sıtkı Koçman University, Turkey \\ ${ }^{2}$ Department of Molecular Biology and Genetics Faculty of Science, Muğla Sitkı Koçman University, Turkey \\ ${ }^{3}$ Department of Medical Biology Faculty of Medicine, Muğla Sitkı Koçman University, Turkey
}

*Corresponding author: Tuba Gökdoğan Edgünlü, Faculty of Medicine Department of Medical Biology, Muğla Sıtkı Koçman University, 4800 Muğla, Turkey.

To Cite This Article: Tuba Gökdoğan Edgünlü, Tunneling Nanotube-Mediated Mitochondrial Transfer: A New Approach to Cell Protection. Am J Biomed Sci \& Res. 2020 - 7(1). AJBSR.MS.ID.001119. DOI: 10.34297/AJBSR.2020.07.001119.

Received: 制 January 16, 2020; Published: 㘹 January 29, 2020

\begin{abstract}
Mitochondria are eukaryotic organelles involved in crucial biochemical processes in cells and owing to their importance in cellular functions; many studies have been conducted to demonstrate the effects of mitochondrial transfer between physically separated cells. Tunneling nanotubes have been denoted as the major route for mitochondrial transfer. Given that mitochondria regulate many cellular processes; perturbation in their internal organization ultimately leads to cellular failure. Interventions by using stem cells have shown that cell injury as a result of mitochondrial dysfunction caused by different insults can be reversed as miscellaneous studies have indicated
\end{abstract}

Keywords: Mitochondrial transfer; Tunneling nanotubes; Mesenchymal stem cells; Cell injury

Abbreviation: BMSCs: Bone marrow stromal cells; CEC: Corneal epithelial cells; CS: Cigarette smoke; ECs: Epithelial cells; EGF: Epidermal growth factor; EGFP: Enhanced green fluorescent protein; EGFR: Epidermal growth factor receptor; HUVECs: Human umbilical vein endothelial cells; iPSCMSCs: Induced pluripotent stem cell-derived mesenchymal stem cells; MCAO: Middle cerebral artery occlusion; MMSCs: Multipotent mesenchymal stem cells; MPs: Membrane protrusions; mTOR: Mammalian target of rapamycin; NSCs: Neural stem cells; OGD: Oxygen-glucose deprivation; OXPHOS: Oxidative phosphorylation; PS: Phosphatidylserine; RCN: Rat cortical neurons; Rot: Rotenone; TNTs: Tunneling nanotubes; WJMSCs: Wharton's jelly-derived mesenchymal stem cells

\section{Introduction}

Many cellular metabolic pathways are dependent on sufficient supply of ATP; NAD; and FADH2 which are produced in the mitochondria; double-membrane eukaryotic organelles containing 16.569 base-pair mtDNA [1]. Although mitochondria are considered as the "powerhouses" of the cell; they have a variety of critical disparate functions by which many cellular processes are regulated such as fatty acid and cholesterol synthesis; amino acid synthesis; management of redox balance; waste management; apoptosis; autophagy and senescence [2-4].

Lynn et al. has proposed that not only mitochondria and chloroplast but also eukaryotic flagellum and mitotic apparatus originated from endosymbiotic organisms [5]. It is considered that early "proto-mitochondria" evolved from alpha-proteobacterium and many genomic and proteomic changes occurred throughout the evolutionary processes [6,7]. Given that mitochondria have crucial functions for cell metabolism; perturbation of their internal integrity may culminate in abnormal cell dynamics which can lead to disturbed cell functioning and even cell death.

\section{Mitochondrial Transfer}

In vitro mitochondrial transfer was first exemplified with an experiment in which mtDNA mutated and depleted A549 cells were rescued by human bone marrow stem cells [8]. It has been demonstrated that mitochondria can be donated intercellularly by means of different mechanisms such as tunneling nanotubes; cell fusion; extracellular vesicles; and mitochondrial extrusion [9]. Recently, many studies have been demonstrated regarding beneficial effects of mitochondrial transfer with both in vitro and 
in vivo studies. Intercellular mitochondrial transfer may result in enhanced cell survival or cellular reprogramming and cell stress may enhance intercellular organelle transport to stressed cells [10]. The latter one has been illustrated by many experimental studies in which stressors stimulated mitochondrial transport towards stimulated cells. Chondrocytes that are stressed by Interleukin-1 beta or mitochondria specific stressors received mitochondria from MSCs [11]. ROT-induced corneal epithelial cells (CECs) enhanced their mitochondrial uptake from MSCs that have functional mitochondria [12]. This result highlights the fact that donor cells must have functional mitochondria to be able to have therapeutic effects on injured cells. Treatment of human umbilical vein endothelial cells (HUVECs) with ethidium bromide resulted in MSC-derived tunneling nanotubes through which mitochondria are transported to injured HUVECs. An intriguing finding has also been detected that phosphatidylserine (PS) domains on HUVECs' cell surfaces were necessary for complete TNT formation. Annexin $\mathrm{V}$; which binds to phosphaditylserine (PS); significantly reduced the number of TNTs but caused insignificant change in the number of membrane protrusions (MPs) [13]. Apparently, PSs on MPs are recognized by MSCs to fully form TNTs (All of the related points summarized in the (Table 1).

\begin{tabular}{|c|c|c|c|c|}
\hline Donor Cell & Recipient Cell & Disease Model & Consequence of Transportation & References \\
\hline $\mathrm{MSCs}^{1}$ & Chondrocytes & $\begin{array}{l}\text { IL-1ß, oligomycin, and rotenone-mediated chon- } \\
\text { drocyte injury. }\end{array}$ & Not mentioned. & [11] \\
\hline MSCs & HUVECs $^{2}$ & $\begin{array}{c}\text { Oxygen-glucose deprivation (OGD) }{ }^{3} \text {-mediated } \\
\text { HUVECs injury. }\end{array}$ & Reduced apoptosis, improved cell viability. & [13] \\
\hline MSCs & $\mathrm{CECs}^{4}$ & $\begin{array}{l}\text { Rotenone (Rot) })^{5} \text {-induced mitochondrial impair- } \\
\text { ment of CECs. }\end{array}$ & $\begin{array}{c}\text { Increased respiratory function, protection } \\
\text { against cell death and proliferation inhibi- } \\
\text { tion. }\end{array}$ & [12] \\
\hline iPSC-MSC ${ }^{6}$ & Bronchial cells & Cigarette Smoke-induced lung damage. & Reverting CS-induced injury. & [28] \\
\hline $\mathrm{BMSCs}^{7}$ & $\begin{array}{l}\text { Alveolar epithelial } \\
\text { cells }\end{array}$ & Sepsis-induced acute lung injury. & Increased ATP production. & [27] \\
\hline MSCs & $\mathrm{NSCs}^{8}$ & Cisplastin-induced neuronal damage. & $\begin{array}{l}\text { Normalized mitochondrial membrane } \\
\text { potential. }\end{array}$ & [14] \\
\hline MSCs & Cardiac myocytes & Not mentioned. & Not mentioned. & [35] \\
\hline MMSCs $^{9}$ & PC12, astrocytes & $\begin{array}{l}\text { Oxygen-glucose deprivation (OGD) astrocytes, } \\
\text { ethidium bromide-treated PC12 cells, simulated } \\
\text { ischemic lesion by middle cerebral artery occlu- } \\
\text { sion (MCAO) }{ }^{10} \text {. }\end{array}$ & $\begin{array}{l}\text { Decreased lactate production and normalized } \\
\text { proliferation in PC12 cell, reduced ischemic } \\
\text { damage. }\end{array}$ & [26] \\
\hline $\mathrm{T} 24$ & RT4 & $\begin{array}{l}\text { Demonstration of invasive capability of non-ma- } \\
\text { lignant RT4 cells after mitochondrial transfer. }\end{array}$ & Enhanced invasive ability. & [32] \\
\hline MMSCs & $\begin{array}{l}\mathrm{RCNs}^{11} \text {, astroglial } \\
\text { cells }\end{array}$ & $\begin{array}{l}\text { Simulated ischemic lesion by middle cerebral } \\
\text { artery occlusion (MCAO). }\end{array}$ & Neuroprotection & [31] \\
\hline WJMSCs ${ }^{12}$ & Osteosarcoma cells & Not mentioned. & $\begin{array}{l}\text { Rescued oxidative phosphorylation (OX- } \\
\text { PHOS) }\end{array}$ & [34] \\
\hline
\end{tabular}

Antineoplastic drugs have detrimental effects on healthy cells and investigators have been researching therapeutic approaches against chemotherapy-induced cell damage. Cisplastin; as a neoplastic drug; induce neuronal cell damage and nasal administrations of MSCs in vivo normalized mitochondrial function and membrane potential [14]. These results imply that mitochondrial transfer can be accomplished in in vivo conditions following cellular dysfunction as a result of perturbation of mitochondrial bioenergetics and specific manipulations that enhance TNT development.

\section{Tunneling Nanotubes (TNTs)-Mediated Intercellular Transport System}

Although many intercellular transport mechanisms have been described so far; one of them has a pronounced involvement in intercellular transport in vitro and in vivo; named as tunneling nanotubes (TNTs). TNTs were first discovered between PC12 cell line and have a diameter range from 20 to $500 \mathrm{~nm}$ mediating cellular communication between cells [15]. TNTs are membrane spanning protrusions formed between physically separated cells and are composed of F-actin and microtubules; the latter was exemplified in macrophages as thin and thick TNTs containing only actin or both actin and microtubules; respectively [16]. However, a recent study demonstrated the co-distribution of actin filaments; intermediate filaments and microtubules in tunneling nanotubes [17]. Following Rustom et al.; TNT configuration was observed in different cell types such as neurons; rat cardiac myocytes; rat kidney; myeloid cells and endothelial progenitor cells [18]. Kumar et al. has illustrated that TNTs are formed between epithelial cell lines and following TEM analyses have revealed that many mitochondria and ribosomes are shuttled in TNTs [19]. Several mechanisms have been proposed in terms of induction of TNT development. Treatment with stressful 
insults culminates in enhanced TNTs. Subsequent analyses have proposed that proto-oncogene p53 expression is an important factor for TNT development. p53-deficient cells were unable to form TNTs.

In addition; EGFR and downstream pathways involving Akt; PI3K and mTOR were shown to be important regulators of TNT formation [20]. These results are consistent with the studies unveiled that mTORC2-mediated signaling pathways have been implicated in actin cytoskeleton organization [21,22]. Furthermore, inhibition of mTOR has led to the reduced TNT development [23]. Activation of EGFR; akin to mTOR; culminated in increased actin filament formation at the apical membrane of the cells which were treated with EGF [24]. Moreover, Wnt/ $\mathrm{Ca}^{2+}$ signaling pathway has been shown to induce TNT formation [25]. Notably, mitochondrial transportation through TNTs is enhanced by overexpression of Miro1 in multipotent mesenchymal stem cells (MMSCs) and intravenous administrations of MMSCs reversed the effects of ischemia on neuronal functions more efficiently than those MMSCs without overexpression of Miro1 [26]. Taken together; these results suggest that miscellaneous mechanisms impact TNT development.

\section{Impacts of Mitochondrial Transfer on in vivo Disease Models}

In vitro studies emerge a number of questions regarding whether mitochondrial transfer is seen in only in vitro conditions or it can be seen in in vivo conditions as well. Islam et al. has noted that bone marrow stromal cells (BMSCs) are able to transfer mitochondria to injured alveolar epithelial cells in an in vivo acute lung injury model [27]. In another study; induced pluripotent stem cell-derived mesenchymal stem cells (iPSC-MSCs) were shown to have beneficial effects on Cigarette-Smoke induced lung damage; whereas bone marrow-derived mesenchymal stem cells (BM-MSCs) are less effective in attenuating lung damage compared to iPSCMSCs in a CS-induced rat model [28]. Additionally, mitochondrial transfer from iPSC-MSCs to epithelial cells (ECs) via TNT alleviates asthmatic inflammation [29]. Similarly, iPSC-MSCs and BM-MSCs can donate mitochondria to cardiomyocytes with doxycyclineinduced damage [30]. Stroke is an important disease owing to its high risk of sequelae. That's why it might has drawn attention to investigate therapeutic approaches.

Babenko et al. illustrated therapeutic effects of MMSCs in the setting of stroke by the study in which neuroprotection has been accomplished following mitochondrial transfer to rat cortical neurons (RCN) [31]. On the other hand, mitochondrial transfer might have adverse effects. Such condition has been demonstrated in a study in which malignant T24 cells enhanced non-malignant RT4 cells' invasive ability by transferring mitochondria through TNTs [32]. Proliferation and invasiveness of cancer cells increased due to recovered oxidative phosphorylation (OXPHOS) followed by mitochondrial transfer [33]. A similar research determined that osteosarcoma cells rescued from impaired oxidative phosphorylation by WJMSCs [34, 35]. It is also possible that chemoresistance can be gained through transfer of mitochondria. Such finding has been observed in a study in which endothelial cells were found to be contributed to chemoresistance development by transferring mitochondria to cancer cells [36].

\section{Conclusion}

As we reviewed here; TNT formation mechanism depends on many signaling pathways, but it remains to be fully elucidated. Understanding this peculiar cellular communication system would increase our chance to develop more efficient therapeutic approaches. Whilst stem cell therapy per se remains to be an important entity; mitochondrial transfer through stem cell donation may serve as a therapeutic treatment in the future.

\section{Conflicts of Interest}

The authors declare no conflict of interest.

\section{References}

1. Patananan AN, Wu TH, Chiou PY, Teitell MA (2016) Modifying the Mitochondrial Genome. Cell Metab 23(5): 785-796.

2. Abate M, Festa A, Falco M, Lombardi A, Luce A, et al. (2020) Mitochondria as playmakers of apoptosis, autophagy and senescence. Semin Cell Dev Biol 98: 139-153.

3. Desagher S, Martinou JC (2000) Mitochondria as the central control point of apoptosis. Trends Cell Biol 10(9): 369-377.

4. Spinelli JB, Haigis MC (2018) The multifaceted contributions of mitochondria to cell ular metabolism. Nat Cell Biol 20(7): 745754 .

5. Sagan L (1967) On the origin of mitosing cells. J Theor Biol 14(3): 255274.

6. Roger AJ, Muñoz-Gómez SA, Kamikawa R (2017) The Origin and Diversification of Mitochondria. Curr Biol 27(21): R1177-R1192.

7. Gabaldón T, Huynen MA (2004) Shaping the mitochondrial proteome. Biochim Biophys Acta 1659(2-3): 212-220.

8. Spees JL, Olson SD, Whitney MJ, Prockop DJ (2006) Mitochondrial transfer between cells can rescue aerobic respiration. Proc Natl Acad Sci 103(5): 1283-1288.

9. Torralba D, Baixauli F, Sánchez-Madrid F (2016) Mitochondria Know No Boundaries: Mechanisms and Functions of Intercellular Mitochondrial Transfer. Front Cell Dev Biol 4: 107.

10. Rogers RS, Bhattacharya J (2013) When cells become organelle donors. Physiology (Bethesda) 28(6): 414-422.

11. Bennett MP, Vivancos-Koopman I, Seewald L, Wells K, Robinette T, et al. (2019) Intercellular mitochondrial transfer from mesenchymal stem cells to stressed chondrocytes. Osteoarthritis and Cartilage 27: 51-52.

12. Jiang D, Gao F, Zhang Y, Wong DS, Li Q, et al. (2016) Mitochondrial transfer of mesenchymal stem cells effectively protects corneal epithelial cells from mitochondrial damage. Cell Death Dis 7(11): e2467.

13. Liu K, Ji K, Guo L, Lu H, Shan P, et al. (2014) Mesenchymal stem cells rescue injured endothelial cells in an in vitro ischemia-reperfusion model via tunneling nanotube like structure-mediated mitochondrial transfer. Microvasc Res 92: 10-18.

14. Boukelmoune N, Chiu GS, Kavelaars A, Heijnen CJ (2018) Mitochondrial transfer from mesenchymal stem cells to neural stem cells protects 
against the neurotoxic effects of cisplatin. Acta Neuropathol Commun 6(1): 139

15. Rustom A, Saffrich R, Markovic I, Walther P, Gerdes HH, et al. (2004) Nanotubular highways for intercellular organelle transport. Science 303(5660): 1007-1010.

16. Onfelt B, Nedvetzki S, Benninger RK, Purbhoo MA, Sowinski S, et al (2006) Structurally distinct membrane nanotubes between human macrophages support long-distance vesicular traffic or surfing of bacteria. J Immunol 177(12): 8476-8483.

17. Resnik N, Erman A, Veranič P, Kreft ME (2019) Triple labelling of actin filaments, intermediate filaments and microtubules for broad application in cell biology: uncovering the cytoskeletal composition in tunneling nanotubes. Histochem Cell Biol 152(4): 311-317.

18. Abounit S, Zurzolo C (2012) Wiring through tunneling nanotubes--from electrical signals to organelle transfer. J Cell Sci 125(5): 1089-1098.

19. Kumar A, Kim JH, Ranjan P, Metcalfe MG, Cao W, et al. (2017) Influenza virus exploits tunneling nanotubes for cell-to-cell spread. Sci Rep 7: 40360.

20. Wang Y, Cui J, Sun X, Zhang Y (2011) Tunneling-nanotube development in astrocytes depends on p53 activation. Cell Death Differ 18(4): 732 742.

21. Angliker N, Rüegg MA (2013) In vivo evidence for mTORC2-mediated actin cytoskeleton rearrangement in neurons. Bioarchitecture 3(4): 113-118.

22. Jacinto E, Loewith R, Schmidt A, Lin S, Rüegg MA, et al. (2004) Mammalian TOR complex 2 controls the actin cytoskeleton and is rapamycin insensitive. Nat Cell Biol 6(11): 1122-1128.

23. Desir S, Dickson EL, Vogel RI (2016) Tunneling nanotube formation is stimulated by hypoxia in ovarian cancer cells. Oncotarget 7(28): 4315043161.

24. Rijken PJ, van Hal GJ, van der Heyden MA, Verkleij AJ, Boonstra J, et al. (1998) Actin polymerization is required for negative feedback regulation of epidermal growth factor-induced signal transduction. Exp Cell Res 243(2): 254-262.

25. Vargas JY, Loria F, Wu YJ, Córdova G, Nonaka T, et al. (2019) The Wnt/ $\mathrm{Ca}^{2+}$ pathway is involved in interneuronal communication mediated by tunneling nanotubes. EMBO J 38(23): e101230.

26. Babenko VA, Silachev DN, Popkov VA, Zorova LD, Pevzner IB, et al. (2018) Miro1 Enhances Mitochondria Transfer from Multipotent Mesenchymal
Stem Cells (MMSC) to Neural Cells and Improves the Efficacy of Cell Recovery. Molecules 23(3): 687.

27. Islam MN, Das SR, Emin MT, Wei M, Sun L, et al. (2012) Mitochondrial transfer from bone-marrow-derived stromal cells to pulmonary alveoli protects against acute lung injury. Nat Med 18(5): 759-765.

28. Li X, Zhang Y, Yeung SC, Liang Y, Liang X, et al. (2014) Mitochondrial transfer of induced pluripotent stem cell-derived mesenchymal stem cells to airway epithelial cells attenuates cigarette smoke-induced damage. Am J Respir Cell Mol Biol 51(3): 455-465.

29. Yao Y, Fan XL, Jiang D, Zhang Y, Li X, et al. (2018) Connexin 43-Mediated Mitochondrial Transfer of iPSC-MSCs Alleviates Asthma Inflammation. Stem Cell Reports 11(5): 1120-1135.

30. Zhang Y, Yu Z, Jiang D, Liang X, Liao S, et al. (2016) iPSC-MSCs with High Intrinsic MIRO1 and Sensitivity to TNF- $\alpha$ Yield Efficacious Mitochondrial Transfer to Rescue Anthracycline-Induced Cardiomyopathy. Stem Cell Reports 7(4): 749-763.

31. Babenko VA, Silachev DN, Zorova LD, Pevzner IB, Khutornenko AA, et al (2015) Improving the Post-Stroke Therapeutic Potency of Mesenchymal Multipotent Stromal Cells by Cocultivation With Cortical Neurons: The Role of Crosstalk Between Cells. Stem Cells Transl Med 4(9): 1011-1020.

32. Lu J, Zheng X, Li F, Yu Y, Chen Z, et al. (2017) Tunneling nanotubes promote intercellular mitochondria transfer followed by increased invasiveness in bladder cancer cells. Oncotarget 8(9): 15539-15552.

33. Caicedo A, Fritz V, Brondello JM, Ayala M, Dennemont I, et al. (2015) MitoCeption as a new tool to assess the effects of mesenchymal stem/ stromal cell mitochondria on cancer cell metabolism and function. Sci Rep 5: 9073.

34. Lin HY, Liou CW, Chen SD, Hsu TY, Chuang JH, et al. (2015) Mitochondrial transfer from Wharton's jelly-derived mesenchymal stem cells to mitochondria-defective cells recaptures impaired mitochondrial function. Mitochondrion 22: 31-44.

35. Plotnikov EY, Khryapenkova TG, Vasileva AK, Marey MV, Galkina SI, et al. (2008) Cell-to-cell crosstalk between mesenchymal stem cells and cardiomyocytes in co-culture. J Cell Mol Med 12(5A): 1622-1631.

36. Pasquier J, Guerrouahen BS, Al Thawadi H, Ghiabi P, Maleki M, eta al. (2013) Preferential transfer of mitochondria from endothelial to cancer cells through tunneling nanotubes modulates chemoresistance. J Transl Med 11(94): 1479-5876. 